\title{
Oclusão Percutânea de Comunicação Interatrial - Ao Gosto do Freguês!
}

\author{
Francisco Chamié
}

A s comunicações interatriais (CIA) correspondem a $10 \%$ dos defeitos congênitos em neonatos e a 30 a $40 \%$ em adultos. ${ }^{1}$ Crianças são geralmente assintomáticas, mas apenas $4 \%$ dos pacientes acima de 40 anos negam sintomas. ${ }^{2}$

\section{Ver página 384}

Embora as CIA sejam, geralmente, bem toleradas, algumas complicações podem surgir na idade adulta, sendo a mais temida o aparecimento da doença vascular pulmonar. Hipertensão arterial pulmonar significativa pode ocorrer em, aproximadamente, 22\% dos adultos com possibilidade de desenvolvimento de hiperresistência vascular pulmonar em $15 \%$ deles. ${ }^{3}$ Outros problemas que podem ocorrer na história natural das CIA são: aumento das cavidades direitas com disfunção do ventrículo direito, dessaturação sistêmica, embolias paradoxais e arritmias atriais, cuja incidência aumenta com a idade.

Iniciada em 1948 por Murray, a correção cirúrgica das CIA do tipo ostium secundum é um procedimento bem estabelecido, seguro, eficaz e com taxa de mortalidade satisfatoriamente baixa. Apresenta, no entanto, complicações (maiores e menores), necessita de tempo de internação mais prolongado, causa dor, provoca cicatrizes visíveis e demanda maior tempo de recuperação quando comparada aos implantes por via percutânea. ${ }^{4}$

A oclusão percutânea, inicialmente descrita por King e Mills, em 1976, pavimentou o caminho para a cardiologia intervencionista moderna dos defeitos congênitos, sendo, talvez, o procedimento mais realizado, atualmente, em nosso meio. O primeiro relato de oclusão percutânea de uma CIA do tipo ostium secundum na literatura nacional surgiu com Haddad et al., em 1996. ${ }^{5}$ Naquela ocasião, predominavam as próteses de duplo disco sem mecanismo centralizador (Duplo Umbrella de Rashkind, CardioSEAL ${ }^{\circledR}$, StarFLEX ${ }^{\circledR}$ e Sideris Buttoned Device $^{\circledR}$ ). Difíceis de manusear, não reposicionáveis e com resultados apenas razoáveis, não encorajaram os intervencionistas brasileiros e seu uso foi limitado a uns poucos casos no Rio de Janeiro e em São Paulo.

O aparecimento de próteses confeccionadas com malha de nitinol (Amplatzer Septal Occluder ${ }^{\circledR}-$ ASO, da AGA Medical Corp., Golden Valley, EUA), em 1997, foi responsável por um aumento exponencial no número de procedimentos em todo o mundo. O ASO era composto por finos fios de nitinol tecidos formando dois discos e uma parte central que funcionava como mecanismo centralizador, o qual se expandia, ocupando inteiramente o defeito e correspondendo ao tamanho nominal da prótese. Era um material muito elástico e com grande memória, permitindo aos dispositivos serem comprimidos em uma bainha longa para recuperar sua forma original, quando exteriorizados. Possuíam retalhos de poliéster suturados em três pontos do dispositivo, com a finalidade de induzir trombose em seu interior. Os ASO apresentavam, ainda, importantes vantagens em relação aos dispositivos anteriores: eram capazes de ocluir defeitos maiores (até $40 \mathrm{~mm}$ ), não necessitavam de borda aórtica para fixação, eram de fácil manuseio, totalmente resgatáveis e reposicionáveis, enquanto presos ao cabo de entrega, permitindo uma curva de aprendizado menor. Em vista disso, rapidamente se transformaram no dispositivo de eleição para a oclusão das CIA do tipo ostium secundum em todo o mundo. Obtiveram a autorização da Food and Drug Administration (FDA) para uso em território americano e são, até hoje, os dispositivos mais referidos na literatura médica internacional. ${ }^{6}$ A experiência brasileira com esse dispositivo é vasta e bem documentada.

Apesar dos excelentes resultados apresentados, associados ao grande número de implantes, surgiram problemas: o aparecimento ou agravamento de cefaleias, arritmias significativas (especialmente os bloqueios atrioventriculares totais - BAVT, e a fibrilação atrial - FA), embolizações tardias do dispositivo, derrames pericár- 
dicos, formação de trombos no disco esquerdo da prótese e as extremamente preocupantes erosões. As complicações gerais do procedimento com o uso dos ASO são em pequeno número $(8,6 \%)$, mas podem ocorrer imediatamente ou vários anos após o implante. ${ }^{7}$

O ASO contém 55\% de seu peso em níquel, material que entra na composição da liga do nitinol. A liberação de níquel do dispositivo se inicia imediatamente após o implante e atinge seu máximo em 1 mês, podendo causar reações alérgicas e tóxicas em pacientes sensíveis. Embora isso não tenha se mostrado um problema de grande magnitude, alguns casos de ocorrência ou agravamento de enxaqueca, em pacientes submetidos ao implante de ASO, têm sido atribuídos a uma reação alérgica ao níquel. Alternativamente, a ativação plaquetária na superfície do dispositivo tem sido proposta como mecanismo desencadeador de enxaqueca e é corroborado por resposta terapêutica ao clopidogrel em alguns casos, mas não em todos. $\mathrm{O}$ estiramento do septo atrial, provocado pela presença do dispositivo, levando à liberação pelos miócitos do peptídeo natriurético atrial, um antagonista vasoativo da vasopressina, foi sugerido como mais uma teoria para o aparecimento de enxaqueca.

A presença de arritmias atriais não é incomum em pacientes com ClA do tipo ostium secundum. Alterações eletrofisiológicas do nó sinusal ou do sistema de condução atrial podem ser devidas à sobrecarga volumétrica ou à distensão atrial em pacientes com CIAs hemodinamicamente significativas e aumento de cavidades direitas. Estudos realizados com eletrocardiografia dinâmica (Holter) não revelaram alterações no ritmo basal em $90 \%$ dos pacientes após o procedimento, mas ocorreram alterações na condução atrioventricular em $7 \%$ dos pacientes estudados.

Outros estudos têm demonstrado que a oclusão percutânea com ASO pode se associar a BAVT, que, por sua vez, pode ocorrer durante o procedimento ou imediatamente após, requerendo monitoração eletrocardiográfica. Apesar disso, a maioria dos casos é transitória, reverte espontaneamente e ocorre principalmente nos defeitos que necessitam das maiores próteses. A incidência de BAVT em crianças oscila em torno de $6 \%$ dos casos. Em adultos, sua incidência é ainda menor, provavelmente devido ao maior tamanho dos pacientes em relação às próteses.

O aparecimento de FA após o procedimento acarreta maior risco de complicações tromboembólicas antes da endotelização completa do dispositivo. A incidência de FA parece aumentar após a oclusão percutânea das CIA do tipo ostium secundum, mas é, também, uma complicação bastante descrita em pacientes não tratados. Esses dados levam alguns autores a especular se a presença de FA, após os procedimentos, seria uma consequência deles ou apenas uma complicação esperada do defeito. Pacientes com FA paroxística preexistente só parecem ser capazes de manter o ritmo sinusal após a oclusão do defeito. Entretanto, isoladamente, apenas o fechamento da $\mathrm{ClA}$, não parece ser capaz de obter esses resultados, e procedimentos eletrofisiológicos associados se fazem necessários. ${ }^{8}$

A embolização dos dispositivos tem sido relatada na maioria das séries de casos numa incidência global de 0,1 a 3,5\%. Ela pode ocorrer de imediato, ou após dias, meses ou anos após o implante. Quando ocorre durante o procedimento, na maioria das vezes, o resgate pode ser realizado capturando o dispositivo com um cateter laço e retirando-o por uma bainha longa de maior calibre em relação a que é utilizada para a entrega da prótese. Passado algum tempo após o implante, o dispositivo torna-se rígido pela formação de trombo em seu interior e não permite mais a captura e retirada percutânea. Nesses casos, a opção é a retirada cirúrgica, com fechamento do defeito eletivamente ou em regime de urgência. ${ }^{9}$ Quando embolizado, o dispositivo tende a se deslocar para o átrio direito, de onde cruza a valva tricúspide e, geralmente, dirige-se para o tronco ou para os ramos pulmonares. Dependendo de sua posição relativa ao eixo do vaso, pode não causar obstrução.

Derrames pericárdicos são descritos na maioria das séries mas sem uma explicação convincente para seu aparecimento. Podem ocorrer imediatamente após o procedimento, como consequência de perfuração cardíaca, ou dias após, sem causa determinada, mas especula-se que possa ser devido à reação imunológica. ${ }^{7}$

A presença de trombos aderidos ao dispositivo, uma complicação temida por suas consequências, parece ser menos frequente nos dispositivos de malha de nitinol do que nos previamente existentes. FA após o procedimento e a presença de aneurisma do septo atrial parecem ser preditores de formação de trombos, mas a dupla antiagregação plaquetária tem se mostrado bastante eficaz na prevenção dessa complicação. ${ }^{10}$

Erosões provocadas pelo ASO são eventos raros, mas potencialmente fatais. São situações de extremo risco para os pacientes, imprevisíveis e podem ocorrer tardiamente. Embora seu mecanismo seja, ainda, desconhecido, a erosão parece estar relacionada à abrasão do tecido cardíaco adjacente pela prótese metálica, que pode ter agentes facilitadores, como defeitos estruturais associados (como na síndrome de Marfan), alterações de espessura e composição teciduais relacionadas à idade, e variações do tecido na capacidade de resistir às forças abrasivas. ${ }^{11} \mathrm{O}$ superdimensionamento dos dispositivos e o contato das bordas da prótese com as paredes aórticas ou atriais, associados à movimentação cardíaca, podem atuar de maneira similar ao mecanismo de uma serra circular.

A espessura dos fios de nitinol aumenta, em determinados tamanhos de dispositivo (11, 18, 26 e $32 \mathrm{~mm}$ ), para manter sua integridade e forma. A maioria das ero- 
sões, nos casos descritos, ocorreram em pacientes que receberam ASO de $26 \mathrm{~mm}$ que é, proporcionalmente, o dispositivo mais rígido para a espessura de fios de nitinol utilizada nas próteses de 26 a $32 \mathrm{~mm} .{ }^{12}$

Em 2004, a incidência estimada de casos de erosão nos Estados Unidos com o uso do ASO era de 0,1\%. Outras publicações referem taxas de erosão variando entre 0,07 e $0,28 \%$ baseadas no número de ASO vendidos ou implantados. Fica claro que esses números são apenas estimativas, uma vez que muitos pacientes não têm seus implantes registrados, e muitos autores não costumam reportar suas complicações, mas, de qualquer modo, a incidência dessa complicação é, felizmente, baixa.

Com relação ao tempo de aparecimento, as erosões podem ocorrer dias após o implante ou em até 6 anos depois. A taxa de mortalidade provocada por erosão é de 0,004 a $0,015 \% .{ }^{13}$ Com relação ao local, as erosões relatadas ocorreram na parte superior (teto) dos átrios direito e esquerdo (com ou sem comprometimento aórtico), na parede aórtica, na parede de ambos os átrios e, em alguns casos, não foi identificado o local da lesão. As erosões parecem ser mais frequentes nos defeitos de grande diâmetro, localizados mais superiormente, sem borda aórtica e nos que recebem próteses superdimensionadas. ${ }^{13}$ Apesar disso, a maioria dos intervencionistas dedicados ao fechamento das $\mathrm{CIA}$ do tipo ostium secundum acredita que a responsável primária pelas erosões seja a movimentação da prótese no septo atrial.

Embora com as causas ainda não totalmente esclarecidas, uma série de recomendações foi feitas por um painel de especialistas, numa tentativa de minimizar os episódios de erosão, tais como: evitar distender o septo com o balão de mensuração utilizando a técnica de oclusão do fluxo (stop-flow), tentar identificar pacientes de risco (defeitos amplos, altos, sem borda aórtica) e seguir de perto pequenos derrames pericárdicos nas primeiras 24 horas após o procedimento. ${ }^{14}$

É consenso que, apesar das complicações relatadas, indubitavelmente, a oclusão percutânea das CIA do tipo ostium secundum com ASO é um procedimento seguro e efetivo. Na esteira do sucesso das próteses AGA, surgiu uma nova geração de dispositivos visando corrigir deficiências ou sugerindo algumas modificações interessantes no desenho das próteses, em sua consistência, na quantidade e espessura dos fios de nitinol, revestimento externo para evitar liberação de níquel e diferentes mecanismos de liberação. Infelizmente, o número de publicações na literatura mundial enfocando esses novos dispositivos é escasso, talvez por não serem aprovados pela FDA. Apesar de a maioria deles ser liberada pela Agência Nacional de Vigilância Sanitária (Anvisa) e estar sendo utilizada em nosso meio por vários especialistas de renome, com excelentes resultados, o número de publicações nacionais sobre o assunto é quase inexistente. Isso torna muito bem-vindo o artigo de Haddad et al., ${ }^{15}$ publicado nesta edição da Revista Brasileira de Cardiologia Invasiva $(\mathbf{R B C I})$, relatando a experiência inicial dos autores com uma nova prótese para oclusão das CIA do tipo ostium secundum.

Para cada um desses novos dispositivos, há vantagens e desvantagens, com relação a aspectos técnicos e do perfil das próteses. Todos eles estão ao nosso alcance e oferecem opções interessantes com segurança, eficácia e reprodutibilidade, de forma que o profissional deve optar por aquele ao qual melhor se adapta. Realmente, hoje em dia, diante das várias opções disponíveis, a escolha das próteses depende do gosto do freguês!

\section{CONFLITO DE INTERESSES}

O autor declara não haver conflito de interesses relacionado a este manuscrito.

\section{REFERÊNCIAS}

1. Hoffman JI, Christianson R. Congenital heart disease in a cohort of 19502 births with long-term follow-up. Am J Cardiol. 1978;42(4):641-7.

2. Borow KM, Karp R. Atrial septal defect: lessons from the past, directions for the future. N Engl J Med. 1990;323(24):1698-700.

3. Craig $\mathrm{Rj}$, Selzer A. Natural history and prognosis of atrial septal defect. Circulation. 1968;37(5):805-15.

4. Butera G, Carminati M, Chessa M, Youssef R, Drago M, Giamberti A, et al. Percutaneous versus surgical closure of secundum atrial septal defect: Comparison of early results and complications. Am Heart J. 2006;151(1):228-34.

5. Haddad JL, Secches A, Finzi L, Nazetta H, Wanderley M, Wanderley $\mathrm{R}$, et al. Oclusão percutânea transvenosa de comunicação interatrial mediante utilização do Buttoned Device. Arq Bras Cardiol. 1996;67(1):17-22.

6. Masura J, Gavora P, Podnar T. Long-term outcome of transcatheter secundum-type atrial septal defect closure using Amplatzer septal occluders. J Am Coll Cardiol. 2005;45(4):505-7.

7. Chessa M, Carminati M, Butera G, Bini RM, Drago M, Rosti $\mathrm{L}$, et al. Early and late complications associated with transcatheter occlusion of secundum atrial septal defect. J Am Coll Cardiol. 2002;39(6):1061-5.

8. Hill SL, Berul Cl, Patel HT, Rhodes J, Supran SE, Cao QL, et al. Early ECG abnormalities associated with transcatheter closure of atrial septal defects using the Amplatzer septal occluder. I Interv Card Electrophysiol. 2000;4(3):469-74.

9. Delaney JW, Li JS, Rhodes JF. Major complications associated with transcatheter atrial septal occluder implantation: a review of the medical literature and the manufacturer and user facility device experience (MAUDE) database. Congenit Heart Dis. 2007;2(4):256-64.

10. Krumsdorf $U$, Ostermayer S, Billinger K, Trepels T, Zadan E, Horvath $\mathrm{K}$, et al. Incidence and clinical course of thrombus formation on atrial septal defect and patent foramen ovale closure devices in 1000 consecutive patients. J Am Coll Cardiol. 2004;43(2):302-9.

11. Crawford GB, Brindis RG, Krucoff MW, Mansalis BP, Carrol JD. Percutaneous atrial septal occluder devices and cardiac erosion: a review of the literature. Catheter Cardiovasc Interv. 2012;80(2):157-67.

12. Amin Z. Echocardiographic predictors of cardiac erosion after Amplatzer septal occluder placement. Catheter Cardiovasc Interv. 2013 Aug 27. [Epub ahead of print] 
13. Diab K, Kenny D, Hijazi ZM. Erosions, erosions and erosions! Device closure of atrial septal defects: How safe is safe? Catheter Cardiovasc Interv. 2012;80(2):168-74.

14. Amin Z, Hijazi ZM, Bass JL, Cheatham JP, Hellenbrand WE, Kleinman CS. Erosion of Amplatzer septal occluder device after closure of secundum atrial septal defects: review of registry of complications and recommendations to minimize future risks. Catheter Cardiovasc Interv. 2004;63(4):496-502.

15. Haddad JL, Novaes GC, Pavão RB, Badran AV, Lemos DC, Lago IM, et al. Oclusão percutânea de comunicação interatrial tipo ostium secundum com prótese Memopart. Rev Bras Cardiol Invasiva. 2013;21(4):384-9. 